\title{
Validação preliminar de um questionário para avaliar as necessidades psicológicas básicas em Educação Física
}

\author{
A. Pires, L. Cid, C. Borrego, J. Alves, C. Silva
}

A teoria da autodeterminação é uma a abordagem psicológica sobre a motivação, que se preocupa com as causas e as consequências da forma como o ser humano regula o seu comportamento. Segundo vários autores, este modelo teórico pode fornecer informações importantes sobre o processo motivacional dos alunos para as aulas de Educação Física, no entanto, em Portugal não existe ainda nenhum instrumento de avaliação das necessidades psicológicas básicas neste contexto. Desta forma, o objectivo principal deste estudo é a validação preliminar da adaptação à Educação Física da versão Portuguesa do Basic Psychological Needs Exercise Scale (BPNESp), determinando as suas qualidades psicométricas iniciais através de uma análise factorial exploratória do modelo que a suporta. Para tal, participaram no estudo 150 alunos $(n=150)$ do $2^{\circ}$ e $3^{\circ} \mathrm{CEB}$, com idades compreendidas entre os 11 e 16 anos $(M=13.39, D P=1.44)$ e com diferentes níveis de prática desportiva. Os resultados obtidos revelaram uma estrutura factorial igual à versão original (12 itens agrupados em 3 factores, com 4 itens em cada factor), que apresentam índices bastante aceitáveis de validade e fiabilidade, o que leva a concluir que este questionário poderá ser utilizado, com um elevado grau de confiança, em futuras investigações que pretendam avaliar as necessidades psicológicas básicas no contexto da Educação Física. Palavras-chave: avaliação psicológica, educação física, teoria da autodeterminação

\section{Preliminary validation of a questionnaire to measure basic psychological needs in Physical Education}

The self-determination theory is a psychological approach to motivation that focuses on causes and consequences of human behavior regulation. According several authors, this theoretical framework could provide important information about the student's motivational process to physical education class, however, in Portugal does not exists any instrument to measure the basic psychological needs in this domain. So, the main propose of this study is the preliminary adaptation to physical education contexts of Basic Psychological Needs Exercise Scale (Portuguese version: BPNESp), and determine their initial psychometrics properties through an exploratory factor analysis. This propose was accomplished with a sample of 150 students $(n=150)$ from de $2^{\text {nd }}$ and $3^{\text {rd }} C E B$, aged from 11 to 16 years $(M=13.39, S D=1.44)$ with different levels of sports practice. Results revealed a factorial structure just like the original model (12 items grouped in 3 factors, with 4 items hitch factor) and presents acceptable values of validity and reliability. Those findings allow us to conclude, that questionnaire can be used in future investigations to measure the basic psychological needs in physical education.

Keywords: psychological assessment, physical education, self-determination theory

Ana Pires. Escola Básica 2/3 da Castanheira do Ribatejo - Agrupamento D. António de Ataíde, Mestranda em Educação Física Escolar da Escola Superior de Desporto de Rio Maior, Portugal.

Luís Cid, Carla Borrego e Carlos Silva. Escola Superior de Desporto de Rio Maior, Centro de Investigação em Desporto, Saúde e Desenvolvimento Humano (CIDESD).

José Alves. Instituto Superior de Ciências da Saúde - Norte, Centro de Investigação em Desporto, Saúde e Desenvolvimento Humano (CIDESD), Portugal.

Endereço para correspondência: Ana Pires, Escola Superior de Desporto de Rio Maior, Av. Dr. Mário Soares, Pavilhão Multiusos, 2040-413 Rio Maior - Portugal.

E-mail: ana_apires@sapo.pt 
De acordo com Roberts (2001), existem pelo menos trinta e duas teorias distintas sobre a motivação, sendo este um dos "tópicos que os investigadores mais têm dedicado o seu tempo e energia" (Biddle \& Mutrie, 2001, p.27). Recentemente, numa revisão da literatura sobre este tema (Kingston, Harwood, \& Spray, 2006), consideraram que as teorias sociocognitivas, entre as quais a teoria da autodeterminação, estão entre as mais populares e contemporâneas abordagens teóricas que têm sido utilizadas para examinar os processos motivacionais no campo da Psicologia do Desporto e do Exercício. De facto, nos últimos anos a Teoria da Autodeterminação (TAD: Deci \& Ryan, 1985), tem sido utilizada como modelo teórico de suporte de diversos estudos, com aplicações em vários contextos, nomeadamente, no desporto (Gagne, Ryan, \& Bargmann, 2003; Reinboth \& Duda, 2006), no exercício (Edmunds, Ntoumanis, \& Duda, 2006; Hagger \& Chatzisarantis, 2008) e na educação física (Ntoumanis, 2001; Standage, Duda, \& Ntoumanis, 2003, 2005).

A TAD (ver figura 1) é uma macro teoria sobre a motivação humana que se preocupa com o desenvolvimento e funcionamento da personalidade em contextos sociais, mais concretamente, com as causas e as consequências do comportamento autodeterminado.

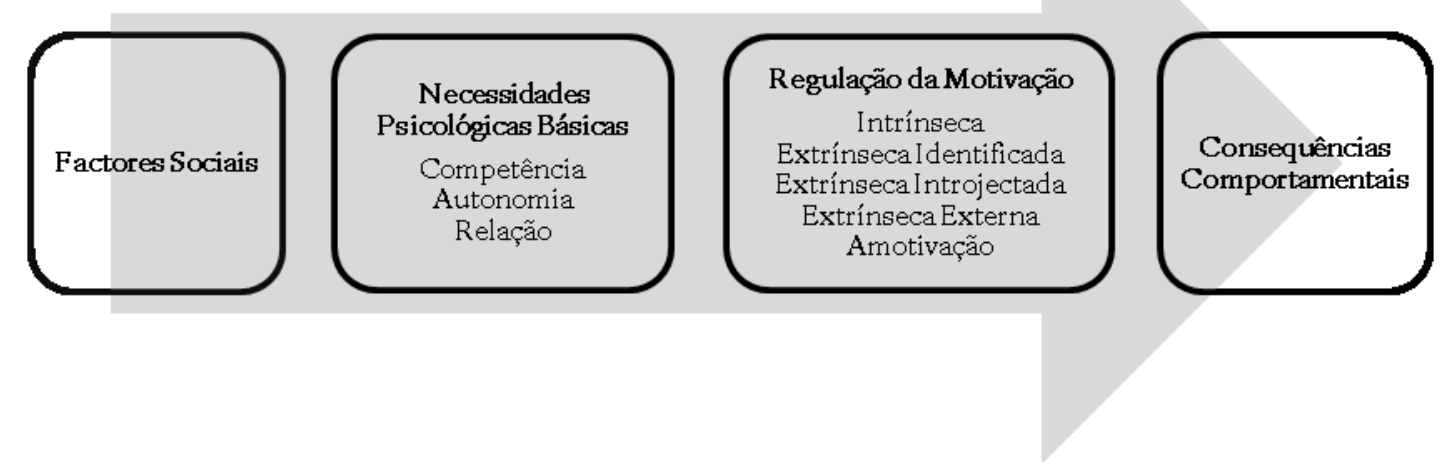

Figura 1. Teoria da Autodeterminação (TAD: Deci \& Ryan, 1985)

(adaptado de Standage, Gillison, \& Treasure, 2007, p.72)

De acordo com os seus autores (Ryan \& Deci, 2000a, 2000b, 2007), esta teoria diz-nos que a motivação do sujeito não está directamente relacionada com os factores do envolvimento social, uma vez que a influência destes (clima das aulas, comportamento dos professores) é mediada pela satisfação de três "componentes fundamentais" (Ryan \& Deci, 2007, p.13), ou seja, as necessidades psicológicas básicas de auto- nomia (capacidade de regular as suas próprias acções), competência (capacidade de eficácia na interacção com o envolvimento) e relação (capacidade de procurar e desenvolver ligações e relações interpessoais). São estas necessidades que vão determinar a regulação do comportamento do sujeito, que assenta num continuum motivacional e oscila entre formas menos e mais auto- 
determinadas do comportamento (controladoras $v s$ autónomas).

Segundo Ryan e Deci (2000b), os sujeitos tendem a participar mais nas actividades quando regulam o seu comportamento para formas mais autodeterminadas: motivação intrínseca (por prazer ou divertimento, sem necessidade de reforço ou recompensa externa) e motivação extrínseca identificada (pela importância pessoal de determinados aspectos, tais como a aprendizagem de novas habilidades). Por oposição, os sujeitos tendem a participar menos nas actividades quando regulam as suas acções para formas menos autodeterminadas: motivação extrínseca introjectada (para evitar sentimentos de culpa ou obter aprovação externa), motivação extrínseca externa (para obter recompensas externas ou evitar punições) e amotivação (falta de motivação e intencionalidade).

Em suma, de acordo com Ryan e Deci (2000a), podemos considerar que a TAD é uma abordagem à motivação alicerçada numa meta teoria que realça a importância dos recursos próprios do ser humano na autoregulação do seu comportamento. Esta passa pela satisfação das necessidades psicológicas básicas de competência, autonomia e relação, pois são elas que estão na base do comportamento autodeterminado (regulação para formas mais intrinsecamente motivadas). Qual é a importância deste facto? A resposta é simples: as pessoas que regulam intrinsecamente a sua motivação, demonstram maior persistência, empenho, esforço e prazer nas actividades que realizam (Ryan \& Deci, 2000b). É por essa razão que a aplicação da TAD ao contexto da educação física (EF) faz todo o sentido e é de extrema pertinência. Segundo Standage, Duda e Ntoumanis (2003), este modelo teórico pode fornecer informações importantes sobre o processo motivacional, nomeadamente, as ligações entre a forma como os alunos regulam o comportamento e o seu compromisso com as actividades realizadas nas aulas de EF e também com a actividade desportiva fora da escola.

De acordo com Standage, Duda e Ntoumanis (2003, 2005), apesar da TAD ter vindo a ser aplicada com sucesso há mais de uma década ao contexto educacional, o mesmo não tem acontecido relativamente ao contexto da educação física escolar, uma vez que os trabalhos nesta área têm sido escassos. Seja como for, alguns estudos recentes aplicados ao contexto da EF (Fernandes, Vasconcelos-Raposo, Lázaro, \& Dosil, 2004; Ntoumanis, 2001, 2005; Standage, Duda, \& Ntoumanis, 2003, 2005) indicam que as formas mais autodeterminados de regulação do comportamento são aquelas que estão relacionadas com as consequências mais positivas, tais como: o esforço, o empenho, a felicidade e a concentração nas aulas. Os alunos que regulam o comportamento desta forma são aqueles que aceitam melhor as tarefas desafiadoras e demonstram intenções mais fortes de praticar actividade física opcional dentro e fora da escola, sendo a satisfação das necessidades psicológicas básicas (em especial da competência), o principal mediador sobre as formas mais autónomas da motivação 
(motivação intrínseca e motivação extrínseca identificada).

Por outro lado, os mesmos autores indicam que as consequências mais negativas (aborrecimento, desilusão, embaraço e infelicidade) estão mais associadas com as formas de regulação menos autodeterminadas. Os estudos de Standage, Duda e Ntoumanis (2005) e Cox e Williams (2008), revelam ainda que os alunos que percepcionam um clima de suporte das suas necessidades psicológicas básicas por parte do professor, são aqueles que têm maiores níveis de satisfação e, consequentemente, mais facilmente regulam o seu comportamento para formas intrinsecamente motivadas.

No entanto, no que se refere apenas à forma como se avaliaram as necessidades psicológicas básicas, só num dos estudos consultados (Ntoumanis, 2005), foi utilizada uma escala de raiz (Basic Need Satisfaction Scale at Work: ver Deci, Ryan, Gagné, Leone, Usunov, \& Kornaheva, 2001), composta por 21 itens que avaliam a percepção de competência (6 itens), de relação (8 itens) e de autonomia (7 itens). Apesar de a escala ter sido modificada, adaptada e validada para o contexto da EF (com recurso a uma análise factorial confirmatória), o autor considerou que os índices de ajustamento encontrados não eram satisfatórios $\left(\mathrm{SB} \chi^{2}=838.6\right.$, $d f=186, p=.000 ; S R M R=.11 ; C F I=$ $.70 ;$ RMSEA $=.10)$. A escala só apresentou níveis aceitáveis de ajustamento $(S R M R=.06 ; C F I=.93 ;$ RMSEA $=$ .06), quando o autor procedeu à eliminação dos itens que estavam redigidos negativamente (3 itens em cada factor).
Relativamente à fiabilidade, os níveis de consistência interna foram considerados aceitáveis, apesar do valor da percepção de autonomia ser abaixo de .70 (competência $\alpha=.66$; autonomia $\alpha$ $=.70$; relação $\alpha=.84$ ).

Ainda no que se refere apenas à avaliação das necessidades psicológicas básicas, nos outros estudos consultados (Cox \& Williams, 2008; Fernandes et al., 2004; Ntoumanis, 2001; Standage, Duda, \& Ntoumanis, 2003, 2005), a estratégia encontrada pelos seus autores, para suprimir a falta de instrumentos específico no contexto da $\mathrm{EF}$, foi a utilização de subescalas adaptadas de outros questionários e/ou o desenvolvimento de itens específicos para esse efeito.

No caso do estudo realizado por Ntoumanis (2001), foram utilizados os 5 itens (modificados para o contexto da EF) da subescala da competência do Intrinsic Motivation Inventory (IMI): desenvolvido originalmente por Ryan (1982) e aplicado ao contexto do desporto por McAuley, Duncan e Tammen (1989), e desenvolvidos dois conjuntos de 2 itens para avaliar a percepção de autonomia e de relação. No entanto, os valores da consistência interna encontrados revelaram-se inadequados em algumas das subescalas (competência $\alpha$ $=.85$; autonomia $\alpha=.43$; relação $\alpha=$ .65). O autor atribuiu este facto ao reduzido número de itens em cada uma delas, o que na sua opinião pode ter conduzido a uma subestimação do valor do alfa de Cronbach.

Por outro lado, Standage et al. (2003), para além de utilizarem também os 5 itens modificados da subesca- 
la da competência do IMI, utilizaram ainda 5 itens modificados de uma das subescalas do Need for Relatedness Scale (NRS) desenvolvida originalmente para o contexto do trabalho (Richer \& Vallerand, 1998), para avaliar a percepção de relação, e outros 5 itens para avaliar a percepção de autonomia, que resultaram do trabalho anterior de Ntoumanis (2001) e de outro estudo não publicado. Os valores da consistência interna encontrados revelaram-se aceitáveis em todas as subescalas (competência $\alpha=$ .85; autonomia $\alpha=.81$; relação $\alpha=$ .81). O mesmo aconteceu noutro estudo dos mesmos autores (Standage et al., 2005), no qual foram utilizados os mesmos instrumentos de medida. Neste caso, também obtiveram resultados de consistência interna muito semelhantes (competência $\alpha=.87$; autonomia $\alpha=.80$; relação $\alpha=.87$ ), apesar de ter sido introduzido um novo item (cotado inversamente) na subescala de avaliação da percepção de autonomia.

No trabalho realizado por Cox e Williams (2008), para avaliar a percepção de competência, foram utilizados 6 itens de uma escala de competência atlética para crianças modificados para o contexto da EF que, segundo os autores, foi desenvolvida por Harter em 1985. Para avaliar a percepção de autonomia, foram utilizados apenas 4 itens do estudo de Standage et al. (2003), uma vez que houve a necessidade de eliminar um dos itens por apresentar uma baixa correlação item-factor. Para avaliar a percepção de relação, foram utilizados 7 itens igualmente modificados para o contexto da EF, da Psychological Sense of School Membership Scale
(PSSMS: Goodenow, 1993). Neste último caso, os autores realizaram uma análise factorial exploratória para validar a escala, e decidiram retirar dois itens por apresentarem baixos pesos factoriais no respectivo factor. No final, os valores da consistência interna encontrados revelaram-se adequados (competência $\alpha=.77$; autonomia $\alpha=$ .69; relação $\alpha=.78$ ).

Por último, num dos primeiros estudos realizados em Portugal (Fernandes et al., 2004), em que se utilizou (entre outras variáveis) a avaliação das necessidades psicológicas básicas dos alunos no contexto da EF, no qual participaram 1099 estudantes dos 14 aos 16 anos de idade, os instrumentos de medida utilizados foram os mesmos do estudo de Ntoumanis (2001), após a sua tradução e adaptação para a língua Portuguesa. Assim sendo, neste trabalho os autores utilizaram os 5 itens da subescala da competência do IMI e utilizaram os dois conjuntos de 2 itens desenvolvidos por Ntoumanis (2001) para avaliar a percepção de autonomia e de relação, obtendo os seguintes valores de consistência interna: competência $\alpha=.86$; autonomia $\alpha=.38$; relação $\alpha=.58$, que são semelhantes aos valores obtidos no estudo de Ntoumanis (2001).

Assim sendo, como em Portugal não existe ainda nenhum instrumento de medida específico para este fim, o objectivo principal deste estudo é a validação preliminar da adaptação para o contexto da Educação Física Escolar, da versão Portuguesa (BPNESp: Moutão, Cid, Leitão, Alves, \& Vlachopoulos, 2009) da Basic Psychological Needs 
Exercise Scale (BPNES: Vlachopoulos \& Michailidou, 2006), determinando as suas qualidades psicométricas iniciais através do recurso à análise factorial exploratória do modelo que a suporta.

\section{MÉTODO}

\section{Amostra}

O nosso estudo contou com a participação de 150 alunos $(n=150)$, de ambos os géneros (80 femininos; 70 masculinos), do $2^{\circ}$ e $3^{\circ}$ Ciclos do Ensino Básico Público (46 do $6^{\circ}$ Ano; 40 do $7^{\circ}$ Ano; 35 do $8^{\circ}$ Ano; 29 do $9^{\circ}$ Ano), com uma média de idades de $13.39 \pm$ 1.44 anos (entre os 11 e 16 anos).

Relativamente à prática de actividade desportiva no âmbito escolar, e para além de todos frequentarem de forma assídua as aulas de EF, podemos dizer que apenas 12 alunos participavam regularmente nas actividades do Desporto Escolar (DE), nas modalidades de Andebol $(n=4)$ e Basquetebol $(n=8)$, às quais dedicavam 1 a 2 treinos por semana, que correspondia a uma média de $57.50 \pm 20.06$ minutos de prática por semana (entre 45 a 90 minutos), sendo a sua experiência neste tipo de actividade, muito variada em termos temporais: de 4 a 48 meses ( $M$ $=14.67, D P=3.19$ ).

No que diz respeito ao seu envolvimento em outras actividades físicodesportivas extra-escolares, 56 alunos afirmaram que praticavam desporto fora da escola (15 Futebol; 1 Hóquei; 18 Natação; 5 Basquetebol; 5 Artes Marciais; 2 Patinagem; 6 Ginástica; 1 Atletismo; 2 Dança; 1 Triatlo). Em média tinham $2.57 \pm 1.06$ treinos por semana (entre 1 a 6 ), que correspondia a uma média de $81.43 \pm 24.58$ minutos de prática semanal (entre 30 a 150). A sua experiência, em termos temporais, variava entre 1 a 120 meses $(M=$ 35.64, $D P=29.52)$.

\section{Instrumentos}

$\mathrm{O}$ instrumento original, designado de Basic Psychological Needs in Exercise Scale (BPNES), foi desenvolvido por Vlachopoulos e Michailidou (2006), e é constituído por 12 itens aos quais se responde numa escala do tipo Likert de 5 níveis, que variam entre 1 ("discordo totalmente") e o 5 ("concordo totalmente"). Os itens agrupam-se posteriormente em 3 dimensões (com 4 itens cada), que reflectem as necessidades psicológicas básicas da teoria da autodeterminação (TAD: Deci \& Ryan, 1985): autonomia, competência e relação.

A versão Portuguesa do questionário (BPNESp) foi traduzida e validada preliminarmente (análise factorial exploratória) por Moutão, Cid, Leitão e Alves (2008), sendo a sua estrutura confirmada posteriormente (análise factorial confirmatória) por Moutão et al. (2009).

Para o presente trabalho foi utilizada uma adaptação para o contexto da Educação Física, da versão portuguesa do BPNESp, que passará a ser designada por BPNPES (Basic Psychological Needs in Physical Education Scale - Questionário de Avaliação das Necessidades Psicológicas Básicas em Educação Física). 


\section{Procedimentos}

Recolha de dados

Após os conselhos executivos das escolas serem informados sobre os objectivos do trabalho e obtido a respectiva autorização para a realização do mesmo, contactou-se, através das respectivas directoras de turma, todos os encarregados de educação dos alunos envolvidos, de modo a obter o consentimento por escrito para que os seus educandos fizessem parte do estudo.

$\mathrm{O}$ instrumento de avaliação foi aplicado sempre em locais e condições semelhantes a todos os participantes, ou seja, em salas de aula e em turmas constituídas no máximo por 25 alunos, onde foram garantidas as condições adequadas para que os indivíduos não se sentissem estranhos com a situação e, ao mesmo tempo, pudessem estar concentrados durante o preenchimento dos questionários.

Para promover a honestidade nas respostas toda a informação foi recolhida de forma anónima. Desta forma, ficou garantida a confidencialidade dos dados, assegurando que os mesmos não seriam, em momento algum, transmitidos individualmente a terceiros.

\section{Adaptação do questionário ao contexto da} Educação Física

O processo de adaptação do questionário para o contexto da EF foi realizado através das seguintes etapas, num processo de validação facial (avaliação do conteúdo dos itens e o seu nível de ajustamento às respectivas dimensões), baseado nas propostas metodológicas utilizadas por diversos autores (New- ton, Duda, \& Yin, 2000; Ntoumanis \& Vazou, 2005; Papaioannou, 1994, 1998; Seifriz, Duda, \& Chi, 1992):

1) Elaboração da $1^{a}$ versão da adaptação do questionário ao contexto da EF: realizada pelos investigadores;

2) Avaliação da $1^{a}$ versão da adaptação: realizada individualmente por 5 professores de Educação Física em exercício pleno da sua actividade profissional. Dos comentários e sugestões apresentados, resultou uma segunda versão da adaptação;

3) Avaliação da $2^{a}$ versão da adaptação: realizada por um painel de 5 especialistas em Psicologia do Desporto (3 com formação inicial em Educação Física e Desporto e 2 com formação inicial em Psicologia), da qual resultou a versão final do instrumento, sendo mantidos os seus 12 itens. Nesta etapa, foi-lhes pedido que associassem os itens às respectivas dimensões (autonomia, relação e competência), e classificassem o seu grau de ajustamento à mesma, através de uma escala que variava entre o 1 ("muito mau ajustamento" e o 5 ("muito bom ajustamento"). Os critérios de retenção dos itens foram: i) avaliação unânime por parte dos especialistas, ii) nível de ajustamento à dimensão 4 ("bom Ajustamento") e 5 ("muito bom ajustamento");

4) Posteriormente, a versão final do questionário foi aplicada a 70 alunos do $2^{\circ}$ e $3^{\circ}$ Ciclos do Ensino Básico para testar a sua adequação ao contexto e compreensibilidade por parte da população alvo (estudo piloto). Uma vez que não se verificaram problemas de maior no que diz respeito à compreensão do conteúdo (valor lexical e semântico) 
dos itens, deu-se por terminada esta etapa. Por último, procedeu-se ainda a uma revisão final do Português (tarefa realizada por 2 professores de português).

\section{Análise Factorial Exploratória (AFE)}

Em primeiro lugar, convém referir que o número de sujeitos utilizados no nosso estudo respeita o rácio de $10: 1$ ( $\mathrm{n}^{\mathrm{o}}$ de sujeitos por cada item do questionário), valor que é recomendado para a realização de uma análise factorial exploratória (Hair, Black, Babin, Anderson, \& Tatham, 2006; Hill \& Hill, 2002; Kahn, 2006; Worthintgton \& Whittaker, 2006). Em termos práticos, com um instrumento de 12 itens, seriam necessários no mínimo 120 sujeitos.

Assim sendo e à semelhança dos procedimentos adoptados na validação preliminar do questionário, quer da versão original (BPNES: Vlachopoulos \& Michailidou, 2006), quer da versão portuguesa (BPNESp: Moutão et al., 2008), recorremos à análise factorial exploratória (AFE), realizada no SPSS 15.0, para determinar o número de factores a reter na solução inicial, o número de itens que lhes estão associados e a consistência interna dos mesmos.

Em termos práticos, a AFE torna possível que uma grande quantidade de variáveis seja reduzida a factores (Leitão, 2002; Moreira, 2004; Pestana \& Gajeiro, 2005), ou seja, ao explorar as correlações entre as variáveis observáveis (itens do questionário), permite o seu agrupamento em dimensões (variáveis latentes - as 3 necessidades psicológicas básicas), estimando o número de factores que são necessários para explicar os dados, bem como, as relações estruturais que os ligam às variáveis observáveis (Maroco, 2007). Segundo Leitão (2002), este tipo de análise (também conhecida por análise das componentes principais) é bastante utilizada quando os investigadores não têm $a$ priori qualquer suposição acerca da natureza da estrutura factorial dos seus dados. No entanto, também é bastante comum mesmo quando existem indicações sobre os factores que são fornecidas pelos modelos (como é o nosso caso).

De acordo com vários autores (Hill \& Hill, 2002; Maroco, 2007; Pestana \& Gajeiro, 2005; Worthington \& Whittaker, 2006), deve existir uma correlação elevada entre as variáveis para que a AFE tenha utilidade na estimação de factores comuns, sendo a medida da adequação da amostragem de KaiserMeyer-Olkin (teste KMO) e o teste de esfericidade de Bartlett, aqueles que são mais utilizados, pois permitem aferir a qualidade das correlações de forma a prosseguir ou não com a análise factorial.

Para tal, é recomendado que o valor do teste de KMO seja superior a .6 e que o valor do teste de Bartlett seja significativo, o que no nosso caso foi cumprido (KMO $=.810$; Teste Bartlett $p=.000)$.

Assim sendo, a estrutura factorial da adaptação do questionário foi examinada através da AFE, utilizando o método de extracção das componentes principais (principal components), seguido da rotação dos factores para que se possa obter uma solução factorial mais 
clara e objectiva, maximizando assim os pesos factoriais dos itens (Brown, 2006). No caso concreto do presente estudo, é aconselhado o método de rotação oblíqua (Promax Rotation), uma vez que, tendo em linha de conta o modelo teórico de suporte (teoria da autodeterminação), pressupõem-se que os factores (autonomia, competência e relação) estejam correlacionados entre si (Henson \& Roberts, 2006; Kahn, 2006; Preacher \& MacCallum, 2003; Worthington \& Whittaker, 2006). De acordo com Brown (2006) e Kahn (2006), o método de rotação oblíquo é sempre a melhor escolha, principalmente quando se trata de modelos multifactoriais.

Seguindo as orientações de diversos autores (Blunch, 2008; Brown, 2006; Hair et al., 2006; Henson \& Roberts, 2006; Kahn, 2006; Preacher \& MacCallum, 2003; Worthington \& Whittaker, 2006), os critérios de determinação dos factores utilizados foram:

1) Critério de Kaiser: Factores com valor próprio igual ou superior a 1 (eigenvalue $\geq 1.0$ );

2) Peso factorial dos itens igual ou superior a .5 (factor loadings $\geq .50$ );

3) Inexistência de itens com pesos factoriais com alguma relevância (factor loadings $>.30$ ) em mais do que um factor. Se isso acontecer e se a diferença entre eles não for significativa (crossloadings $\leq .15$ ), o item deve ser eliminado;

4) A percentagem da variância explicada pelos factores retidos deve ser no mínimo de pelo menos $40 \%$;
5) A consistência interna do factor deve ser igual ou superior a .70 (alfa de Cronbach $\geq .70$ );

6) A consistência interna do factor não deve aumentar se o item for eliminado;

7) Só devem ser retidos os factores com pelo menos 3 itens.

Esta metodologia, que engloba a maioria dos critérios mencionados, tem sido utilizada em Portugal por diversos autores no processo de validação de instrumentos de avaliação na área da Psicologia aplicada ao Desporto e ao Exercício (Borrego \& Alves, 2006; Cid, Alves, \& Dosil, 2008a; Cid, Leitão, \& Alves, 2008b; Fernandes, Lázaro, \& Vasconcelos-Raposo, 2005; Fonseca \& Brito; 2001a, 2001b, 2005; Fonseca \& Fox, 2002; Lourenço \& Alves, 2003; Serpa, 1996).

\section{APRESENTAÇÃO E DISCUSSÃO DOS RESULTADOS}

De acordo com o quadro 1, referente à análise descritiva dos resultados, podemos verificar que, para além dos sujeitos não terem utilizado todos os níveis de resposta disponíveis nos itens 9 e 10, o valor médio das respostas a cada um dos itens variou entre $2.99 \pm$ 1.01 (item 6) e $4.31 \pm .89$ (item 5).

Através da análise do quadro 1, podemos ainda constatar que as respostas a diversos itens $(1,2,3,4,5,8,11)$, não têm uma distribuição normal univariada, uma vez que o valor estandardizado (valor Z) das medidas de assimetria (skewness) e achatamento (kurtosis) se situam fora dos intervalos -1.96 e 1.96 (para $p<.05$ ). 
Quadro 1

Análise descritiva das respostas aos itens do BPNPESp

\begin{tabular}{ccccccc}
\hline Item & $\begin{array}{c}\text { Mín.- } \\
\text { Máx. }\end{array}$ & M \pm SD & Assimetria & Valor Z & Achatamento & Valor Z \\
\hline Item1 & $1-5$ & $3.83 \pm .81$ & -.598 & -3.02 & .530 & 1.35 \\
Item2 & $1-5$ & $4.27 \pm .87$ & -1.442 & -7.28 & 2.681 & 6.80 \\
Item3 & $1-5$ & $3.69 \pm .93$ & -.969 & -4.89 & 1.420 & 3.60 \\
Item4 & $1-5$ & $3.62 \pm .87$ & -.467 & -2.36 & .421 & 1.07 \\
Item5 & $1-5$ & $4.31 \pm .89$ & -1.457 & -7.36 & 2.398 & 6.09 \\
Item6 & $1-5$ & $2.99 \pm 1.01$ & -.250 & -1.26 & -.363 & -.92 \\
Item7 & $1-5$ & $3.27 \pm .86$ & -.357 & -1.80 & .567 & 1.44 \\
Item8 & $1-5$ & $4.19 \pm .92$ & -1.286 & -6.49 & 1.800 & 4.57 \\
Item9 & $2-5$ & $3.55 \pm .72$ & .163 & .82 & -.282 & -.72 \\
Item10 & $2-5$ & $3.83 \pm .80$ & -.253 & -1.28 & -.397 & -1.01 \\
Item11 & $1-5$ & $4.29 \pm .91$ & -1.378 & -6.99 & 1.953 & 4.96 \\
Item12 & $1-5$ & $3.27 \pm .84$ & -.271 & -1.37 & .696 & 1.77 \\
\hline
\end{tabular}

Assim sendo, segundo Pestana e Gajeiro (2005) e Maroco (2007), os itens mencionados apresentam uma distribuição assimétrica negativa (enviesada à direita - predominância dos valores mais elevados da variável) e leptocúrtica (menos achatada - valores mais concentrados), revelando que os participantes apresentam uma tendência para valorizar os itens do questionário. Este facto, parece demonstrar que é atribuída importância à satisfação das 3 necessidades psicológicas básicas.

No quadro 2 são apresentados os resultados relativos à solução inicial da estrutura do questionário que foram encontrados com o recurso à AFE, onde são indicados apenas os pesos factoriais (factor loadings) relevantes e considerados como valor mínimo para poderem ser interpretados, ou seja, .30 (Hair et al., 2006; Kahn, 2006; Worthington \& Whittaker, 2006).

Como podemos observar, no quadro 2 são indicados os resultados das comunalidades (communalities - proporção da variância de cada item que é explicada pelo conjunto dos 3 factores extraídos), da matriz de configuração (pattern matrix - que indica a contribuição única de cada item para o factor) e da matriz de estrutura (structure matrix - que para além de indicar a contribuição de cada item para o factor, tem em linha de conta também a relação existente entre os factores) (Cramer, 2003; Hair et al., 2006). Apesar de não ser consensual qual das matrizes deva ser utilizada, é a "matriz de configuração aquela que mais frequentemente é interpretada $e$ reportada na investigação aplicada" (Brown, 2006, p. 33). Segundo os mesmos autores, os resultados da 
matriz de estrutura tendem a ser sobrestimados à medida que as correlações entre factores aumentam. Assim sendo, iremos optar por analisar apenas a matriz de configuração, na medida em que as correlações entre os 3 factores, segundo Pestana e Gajeiro (2005) são baixas (situam-se entre .20 e .40 Relação-Competência: $r=.34$; RelaçãoAutonomia: $r=.27$; CompetênciaAutonomia: $r=.25$ ).

Quadro 2

Análise factorial exploratória (com rotação oblíqua Promax) do BPNPESp

\begin{tabular}{|c|c|c|c|c|c|c|c|}
\hline \multirow{2}{*}{ Itens } & \multirow{2}{*}{ Comunalidades } & \multicolumn{3}{|c|}{ Matriz de Configuração } & \multicolumn{3}{|c|}{ Matriz de Estrutura } \\
\hline & & Factor 1 & Factor 2 & Factor 3 & Factor 1 & Factor 2 & Factor 3 \\
\hline Item 1 & .62 & & .84 & & & .76 & \\
\hline Item 2 & .77 & .85 & & & .87 & .35 & \\
\hline Item 3 & .59 & & & .70 & .30 & .34 & .75 \\
\hline Item 4 & .65 & & .78 & & .35 & .80 & \\
\hline Item 5 & .83 & .93 & & & .91 & & \\
\hline Item 6 & .62 & & & .80 & & & .76 \\
\hline Item 7 & .66 & & .77 & & .30 & .80 & .31 \\
\hline Item 8 & .70 & .81 & & & .82 & .36 & \\
\hline Item 9 & .44 & & .48 & .36 & & .57 & .48 \\
\hline Item 10 & .59 & & .76 & & & .77 & \\
\hline Item 11 & .85 & .93 & & & .92 & & .31 \\
\hline \multirow[t]{2}{*}{ Item 12} & .68 & & & .84 & & & .81 \\
\hline & & $\begin{array}{l}\text { Fac } \\
\text { (Rel }\end{array}$ & $\begin{array}{l}\text { or } 1 \\
\text { ção) }\end{array}$ & $\begin{array}{r}\text { Fac } \\
\text { (Comp }\end{array}$ & $\begin{array}{l}\text { or } 2 \\
\text { tência) }\end{array}$ & $\begin{array}{r}\text { Fac } \\
\text { (Auto }\end{array}$ & $\begin{array}{l}\text { or } 3 \\
\text { omia) }\end{array}$ \\
\hline \multicolumn{2}{|c|}{ Valor Próprio } & \multicolumn{2}{|c|}{4.39} & \multicolumn{2}{|c|}{1.93} & \multicolumn{2}{|c|}{1.66} \\
\hline \multicolumn{2}{|c|}{ \% Variância } & \multicolumn{2}{|c|}{36.58} & \multicolumn{2}{|c|}{16.07} & \multicolumn{2}{|c|}{13.86} \\
\hline
\end{tabular}

A solução inicial preconizada pela AFE, de uma forma geral, apresenta uma estrutura concordante com o modelo original para o contexto do exercício (Vlachopoulos \& Michailidou, 2006). De facto, o resultado da análise, realizada à adaptação do questionário para o contexto da Educação Física (BPNPES), dá suporte ao modelo teórico, uma vez que o agrupamento dos itens indica uma estrutura de 3 factores com valores próprios acima de 1 , nomeadamente, 4.39 para o factor "Relação" (itens 2, 5, 8, 11), 1.93 para o factor "Competência" (itens 1, 4, 7, 10) e 1.66 para o factor "Autonomia" (itens $3,6,9,12$ ), que justificam no seu conjunto $66.51 \%$ da variância total dos resultados. De acordo com Hair et al. (2006), este valor é considerado 
muito satisfatório para os estudos na área das ciências sociais.

Relativamente aos resultados das comunalidades, todas atingiram valores bastante aceitáveis (Hair et al., 2006: acima de .50), o que indica que uma boa parte da variância dos resultados de cada item é explicada pela solução factorial encontrada. A única excepção é o item 9 (comunalidade $=.44$ ), cujo valor indica que existe uma fraca correlação do item 9 com os 3 factores da solução encontrada, o que é um primeiro sinal de que a sua eliminação deve ser considerada. No entanto, alguns autores (Worthington \& Whittaker, 2006), só colocam essa hipótese se os valores forem inferiores a .40 (o que não se verifica neste caso).

No que respeita aos pesos factoriais dos itens nos respectivos factores, e tendo em linha de conta os resultados da matriz de configuração do quadro 2 (embora os resultados da matriz de estrutura sejam semelhantes), todos apresentam valores acima de .50 , que foi o valor critério estabelecido, pese embora o facto de que, segundo Hair et al. (2006), para uma amostra com 150 sujeitos possamos considerar como significativos todos os pesos factoriais acima de .45. Assim, e com excepção do item 9 (peso factorial $=.36-$ o que é um segundo sinal de que se deve ter em consideração a eliminação deste item), todos os restantes têm pesos factoriais que variam entre .70 e .93 (factor "Relação": entre .81 e .93; factor "Competência": entre .76 e .84; factor "Autonomia": entre .70 e .84), o que é considerado excelente (Tabachnick \& Fidell, 1989). Vários autores (Hair et al., 2006; Kahn, 2006; Worthington \& Whittaker, 2006) indicam.30 como valor mínimo para que um item seja retido, cabendo essa decisão ao investigador (Tabachnick \& Fidel, 1989). Para tal, deve ser levado em linha de conta o seu interesse em manter ou não as variáveis (Kahn, 2006), e, principalmente, o modelo teórico subjacente (Henson \& Roberts, 2006).

Por último, ao analisar os pesos factoriais dos itens em todos os factores, verificamos a existência de um cross-loading do item 9, ou seja, este item apresenta um peso factorial relevante em dois factores (Autonomia $=$ .36; Competência $=.48$ ), exibindo o valor mais baixo no factor onde em teoria deveria ter mais peso (autonomia). Seguindo as orientações de Brown (2006) e Worthington e Whittaker (2006), reflectidas nos critérios anteriormente estabelecidos, a eliminação dos itens com cross-loadings deve ser equacionada, especialmente nos casos em que a diferença entre pesos factoriais é inferior a .15 , como se verifica no caso do item 9. No entanto, Worthington e Whittaker (2006), também aconselham alguma prudência em usar este critério para justificar a eliminação dos itens até que seja encontrada uma solução final. Para alem disto, se analisarmos a matriz de estrutura, verificamos, apesar de se manter o crossloading, que o item 9 mantém um peso factorial significativo no factor onde é suposto pertencer ( .48$)$. No presente estudo, tudo indica que existe uma forte relação deste item com o factor "Competência", o que leva a crer que 
os sujeitos ao responderem a esta questão a interpretam como tal.

Ao analisarmos os resultados do quadro 3, verificamos que o alfa de Cronbach apresenta bons valores de consistência interna nos 3 factores (Hill \& Hill, 2000; Hair et al, 2006): factor "Relação" ( $\alpha=.90$ - muito boa), factor "Competência" ( $\alpha=.81$ - boa) e factor "Autonomia" ( $\alpha=.68$ - razoável).

Apesar de na generalidade dos casos se estabelecer o valor.70 como critério para uma razoável consistência interna, podemos aceitar valores até.60, em especial se estamos a realizar uma análise exploratória (Hair et al., 2006). Esta não deixa de ser mais uma chamada de atenção para a necessidade de uma reflexão sobre o factor "Autonomia”, em especial sobre a adequabilidade do item 9, uma vez que a sua eliminação contribui para o aumento da consistência interna do factor, ainda que de forma muito ligeira (ver quadro $3)$.

Quadro 3

Análise da consistência interna do BPNPES

\begin{tabular}{ccc}
\hline & Correlação Item-Factor & Alfa se Item Eliminado \\
\hline Relação $(\alpha=.90)$ & .78 & .88 \\
Item2 & .82 & .86 \\
Item5 & .70 & .90 \\
Item8 & .84 & .86 \\
Item11 & & \\
\hline Competência $(\alpha=.81)$ & .58 & .78 \\
Item1 & .68 & .73 \\
Item4 & .66 & .74 \\
Item7 & .59 & .78 \\
Item10 & & \\
Item9 & .53 & .58 \\
Item6 & .56 & .64 \\
\hline Autonomia $(\alpha=.68)$ & .44 & .69 \\
\hline
\end{tabular}

Por outro lado, as correlações entre os itens e os respectivos factores também podem ser consideradas de moderadas a altas (Pestana \& Gajeiro, 2005) e com excepção do item $9(r=.35-$ correlação baixa) todas as correlações variam entre.44 e .84. Inclusivamente, segundo Hair et al. (2006), todas as correlações item-factor acima de .50 podem ser consideradas como um bom 
sinal de consistência interna, e como se pode observar, na generalidade (10 dos 12 itens) estes resultados excedem esse valor.

Em suma, se analisarmos os resultados apresentados, quer da análise factorial exploratória, quer da consistência interna, à luz dos critérios de determinação dos factores estabelecidos, podemos afirmar que a adaptação do questionário ao contexto da educação física possui excelentes qualidades psicométricas iniciais. E, assim sendo, na nossa opinião, a sua estrutura deve ser mantida de acordo com o modelo original (Vlachopoulous \& Michailidou, 2006), que está subjacente à teoria da autodeterminação (Deci \& Ryan, 1985).

No entanto, dados os problemas encontrados com o item 9, nomeadamente: peso factorial abaixo no factor "Autonomia" ( .36); existência de crossloading com o factor "Competência" com um peso factorial de .48 (diferença inferior a .15); a consistência interna do factor "Autonomia" aumenta ligeiramente se o item for eliminado (passa de .68 para .69); a baixa correlação item/factor ( .35 ), podermos equacionar a hipótese de eliminação do item em questão. Esta foi a solução encontrada pelos autores da versão Portuguesa do questionário para o contexto do exercício, uma vez que o mesmo item 9, também apresentou alguns problemas (ver Moutão et al., 2008, 2009).

Na nossa opinião, a solução de eliminação do item só deve ser fortemente equacionada depois da realização da análise factorial confirmatória (próxima etapa da validação do questionário), caso se verifique que os mesmos problemas persistem.

Por isso, nesta fase preliminar, propomos apenas a revisão do valor semântico-lexical do item - "... as actividades que realizo são aquelas com que mais me identifico", alterando-o para "... as actividades que realizo representam bem aquilo que eu quero fazer", uma vez que a primeira forma não está a ser compreendida pelos alunos como uma questão que avalia exclusivamente a autonomia, e por isso é que apresenta também uma correlação elevada com a dimensão da competência.

\section{CONCLUSÕES}

Tendo em consideração o objectivo proposto para este trabalho, sobre a validação preliminar da adaptação para o contexto da Educação Física da versão Portuguesa da "Basic Psychological Needs in Exercise Scale" (BPNPES), que nesta adaptação ao contexto da educação física, passará a ser designada por "Questionário de Avaliação das Necessidades Psicológicas Básicas em Educação Física - Basic Psychological Needs in Physical Education Scale" (BPNPES - ver apêndice), podemos concluir, que as suas qualidades psicométricas iniciais comprovam a adequação da adaptação efectuada, demonstrando que a sua estrutura factorial possui índices bastante aceitáveis de validade e de fiabilidade. Assim sendo, parece-nos razoável afirmar que a BPNPES poderá ser utilizada, com alguma confiança, na avaliação das necessidades psicológicas básicas dos alunos no contexto da educação física escolar. No entanto, dados os problemas encontrados com o item 9, 
consideramos fundamental a continuação dos estudos que aprofundem a validade factorial deste instrumento de medida. Por isso, sugerimos que a próxima etapa seja a confirmação do modelo através da análise factorial confirmatória (que se encontra já em fase de preparação), na qual deverá ser testada a nova versão proposta para o item 9.

\section{REFERÊNCIAS}

Biddle, S., \& Mutrie, N. (2001). Psychology of physical activity: Determinants, wellbeing and interventions. London: Routledge, Taylor \& Francis Group.

Blunch, N. (2008). Introduction to structural equation modelling using SPSS and AMOS. London: Sage.

Borrego, C., \& Alves, J. (2006). Como avaliar a satisfação dos atletas com as experiências desportivas? Tradução e adaptação do questionário Athlete Satisfaction Questionnaire (ASQ) para Português. Desporto. Investigação \& Ciência, 5, 63-82.

Brown, T. (2006). Confirmatory factor analysis for applied research. New York: The Guiford Press.

Cid, L., Alves, J., \& Dosil, J. (2008a). Tradução e validação da versão portuguesa da Escala de Actitudes hacia la Actividad Física y el Deporte (EAFDp). In A. Noronha, L. Almeida, M. Gonçalves, S. Martins, V. Ramalho (Eds.), Actas da XIII conferência internacional de avaliação psicológica: Formas e contextos. Braga: Psiquilíbrios Edições (CD-Rom).

Cid, L., Leitão, J., \& Alves, J. (2008b). Tradução e validação da versão portuguesa da Goal Orientation in Exercise Scale (GOESp). In A. Noronha, L. Almeida, M. Gonçalves, S. Martins, V. Ramalho (Eds.), Actas da XIII conferência interna- cional de avaliação psicológica: Formas e contextos. Braga: Psiquilíbrios Edições (CD-Rom).

Cox, A., \& Williams, L. (2008). The roles of perceived teacher support, motivational climate, and psychological need satisfation in students' physical education motivation. Journal of Sport \& Exercise Psychology, 30, 222-239.

Cramer, D. (2003). Advanced quantitative data analysis. Maidenhead: Open University Press.

Deci, E., \& Ryan, R. (1985). Intrinsic motivation and self-determination in human behavior. New York and London: Plenum Press.

Deci, E., Ryan, R., Gagné, M., Leone, D., Usunov, J., \& Kornaheva, B. (2001). Need satisfaction, motivation, and well-being in the work organizations of a former Eastern Bloc country. Personality and Social Psychology Bulletin, 27, 930-942.

Edmunds, J., Ntoumanis, N., \& Duda, J. (2006). A Test of self-determination theory in the exercise domain. Journal of Applied Social Psychology, 36 (9), 22402265.

Fernandes, H. M., Vasconcelos-Raposo, J., Lázaro, J., \& Dosil, J. (2004). Validación y aplicación de modelos teóricos motivacionales en el contexto de la educación física. Cuadernos de Psicología del Deporte, 4(1-2), 67-89.

Fernandes, H. M., Lázaro, J., \& Vasconcelos-Raposo, J. (2005). Razões para a não prática desportiva em adultos. Estudo comparativo entre a realidade rural e urbana. Motricidade, 1(2), 106114.

Fonseca, A., \& Brito, A. (2001a). Estudo exploratório e confirmatório à estrutura factorial da versão portuguesa do Perception of Sucess Questionnaire 
(POSQ). Revista Portuguesa de Ciências do Desporto, 1(3), 61-69.

Fonseca, A., \& Brito, A. (2001b). Propriedades psicométricas da versão portuguesa do Intrinsic Motivation Inventory (IMIp) em contextos de actividade física e desportiva. Análise Psicológica, 19(1), 59-76.

Fonseca, A., \& Brito, A. (2005). A questão da adaptação transcultural de instrumentos para avaliação psicológica em contextos desportivos nacionais: $\mathrm{O}$ caso do Task and Ego Orientation in Sport Questionnaire (TEOSQ). Psychologica, 39, 95-118.

Fonseca, A., \& Fox, K. (2002). Como avaliar o modo como as pessoas se percebem fisicamente? Um olhar sobre a versão Portuguesa do Physical SelfPerception Profile (PSPP). Revista Portuguesa de Ciências do Desporto, 2(5), 1123.

Gagne, M., Ryan, R. M., \& Bargmann, K. (2003). Autonomy support and need satisfaction in the motivation and wellbeing of gymnasts. Journal of Applied Sport Psychology, 15, 372-390.

Goodenow, C. (1993). The psychological sense of school membership among adolescents: Scale development and educational correlates. Psychology in the Schools, 30, 79-90.

Hagger, M., \& Chatzisarantis, N. (2008). Self-determination theory and the psychology of exercise. International Review of Sport and Exercise Psychology, 1 (1), 79103.

Hair, J., Black, W., Babin, B., Anderson, R., \& Tatham, R. (2006). Multivariate data analysis ( $6^{\mathrm{a}}$ edição). New Jersey: Pearson Educational, Inc.

Henson, R., \& Roberts, J. (2006). Use of exploratory factor analysis in published research. common erros and some comment on improved practice. Educa- tional and Psychological Measurement, 66(3), 393-416.

Hill, M., \& Hill, A. (2000). Investigação por questionário. Lisboa: Edições Sílabo.

Kahn, J. (2006). Factor analysis in counseling psychology - Research, trainning, and practice: Principles, advances and applications. The Counseling Psychologist 34(5), 684-718.

Kingston, K., Harwood, C., \& Spray, C. (2006). Contemporary approaches to motivation in sport. In $\mathrm{H}$. Melallieu (Ed.), Literature reviews in sport psychology (pp. 159-197). New York: Nova Science Publishers.

Leitão, C. (2002). Metodologia de investigação em educação física e desporto: Estatística multivariada e introdução à análise factorial. Vila Real: SDE - UTAD.

Lourenço, A., \& Alves, J. (2003). Tradução e adaptação do Questionário de Motivação para o Exercício (Exercise Motivation Questionnaire - EMI-2). Desporto, Investigação \& Ciência, 2, 3-11.

McAuley, E., Duncan, T., \& Tammen, V. (1989). Psychometric properties of the intrinsic motivation inventory in a competitive sport setting: A confirmatory factor analysis. Research Quarterly for Exercise and Sport, 60, 48-58.

Maroco, J. (2007). Análise estatística com utilização do SPSS ( $3^{\text {a }}$ ediçãa). Lisboa: Edições Sílabo.

Moreira, J. (2004). Questionários: Teoria e prática. Coimbra: Livraria Almedina.

Moutão, J., Cid, L., Leitão, J., \& Alves, J. (2008). Tradução e validação preliminar da versão Portuguesa do Basic Psychological Needs in Exercise Scale. In J. Díaz, I. Díaz \& J. Dosil (Eds.), La psicología del deporte en iberoamérica: Actas del $2^{\circ}$ Congreso de la Sociedad Iberoamericana de Psicología del Deporte (p. 314). Torrelavega, Espanha. 
Moutão, J., Cid, L., Leitão, J., Alves, J., \& Vlachopoulos, S. (2009). The measurement of basic psychological needs in exercise: Confirmatory factor analysis of the Basic Psycological Needs in Exercise Scale (BPNES-P) - Portuguese Version. In A. Baria, H. Nabli, M. Madani, A. Essieedali, M. Aragon \& A. Ouartassi (Eds.), Book of Abstracts 12th World Congress of Sport Psychology ( $\mathrm{p}$. 187). Marrakesh, Marroco.

Newton, M., Duda, J., \& Yin, Z. (2000). Examination of the psychometric properties of the Perceived Motivational Climate in Sport Questionnaire-2 in a sample of female athletes. Journal of Sport Sciences, 18, 275-290.

Ntoumanis, N. (2001). A selfdetermination approach to the understanding of motivation in physical education. British Journal of Educational Psychology, 71, 225-242.

Ntoumanis, N. (2005). A prospective study of participation in optional school psysical education using a selfdetermination theory framework. Journal of Educational Psychology, 97(3), 444453.

Ntoumanis, N., \& Vazou, S. (2005). Peer motivational climate in youth sport: Measurement development and validation. Journal of Sport \& Exercise Psychology, 27, 432-455.

Papaioannou, A. (1994). Development of a questionnaire to measure achivement orientations in physical education. Research Quarterly for Exercise and Sport, 65(1), 11-20.

Papaioannou, A. (1998). Student's perceptions of the physical education class environment for boys and girls and the perceived motivational climate. Research Quaterly for Exercise and Sport, 69(3), 267-275.
Pestana, M., \& Gajeiro, J. (2005). Análise de dados para ciências sociais: A complementaridade do SPSS. Lisboa: Edições Sílabo.

Preacher, K., \& MacCallum, R. (2003). Repairing Tom Swift's electric factor analysis machine. Understanding Statistics, 2(1), 13-43.

Reinboth, M., \& Duda, J. (2006). Perceived motivacional climate, need satisfaction and indices of well-being in team sports: A longitudinal perspective. Psychology of Sport and Exercise, 7, 269-286.

Richer, S., \& Vallerand, R. (1998). Construction et validation de L'échelle du Sentiment d'Appartenance. Revue Européene de Psychologie Appliquée, 48, 129137.

Roberts, G. (2001). Understanding the dynamics of motivation in physical activity: The influence of achievement goals on motivational processes. In G. Roberts (Ed.), Advances in motivation in sport and exercise (pp. 1-50). Champaign-Illinois: Human Kinetics.

Ryan, R. (1982). Control and information in the intrapersonal sphere: An extension of cognitive evaluation theory. Journal of Personality and Social Psycho$\log y, 43,450-461$.

Ryan, R., \& Deci, E. (2000a). Selfdetermination theory and the facilitation of intrinsic motivation, social development and well-being. American Psychologist, 55 (1), 68-78.

Ryan, R., \& Deci, E. (2000b). Intrinsic and extrinsic motivations: Classic definitions and new directions. Contemporary Educational Psychology, 25, 54-67.

Ryan, R. \& Deci, E. (2007). Active human nature: Self-determination theory and the promotion and maintenance of sport, exercise and health. In M. Hagger \& N. Chatzisarantis (Eds.), Intrinsic motivation and self-determination in exerci- 
se and sport (pp. 1-19). ChampaignIllinois: Human Kinetics.

Seifriz, J., Duda, J., \& Chi, L. (1992). The relationship of perceived motivational climate to intrinsic motivation and beliefs about success in basketball. Journal of Sport \& Exercise Psychology, 14, 375-391.

Serpa, S. (1996). Motivação para a prática desportiva: Validação preliminar do Questionário de Motivação para as Actividades Desportivas (QMAD). In F. Sobral \& A. Marques (Eds.), FAC$D E X$ - Desenvolvimento somato-motor e factores de excelência desportiva nas populações escolares portuguesas (pp. 89-97). Lisboa: Ministério Educação.

Standage, M., Duda, J., \& Ntoumanis, N. (2003). A model of contextual motivation in physical education: Using constructs from self-determination and achievement goal theories to predict physical activity intentions. Journal of Educational Psychology, 95(1), 97-110.

Standage, M., Duda, J., \& Ntoumanis, N. (2005). A test of self-determination theory in school physical education. British Journal of Educational Psychology, 75, 411-433.

Standage, M., Gillison, F., \& Treasure, D. (2007). Self-determination and motivation in physical education. In $\mathrm{M}$. Hagger \& N. Chatzisarantis (Eds.), Intrinsic motivation and selfdetermination in exercise and sport (pp. 71-85). Champaign-Illinois: Human Kinetics.

Tabachnick, B., \& Fidell, L. (1989). Using multivariate statistics ( $2^{\mathrm{a}}$ edição). New York: Harper Collins Publishers.

Vlachopoulos, S., \& Michailidou, S. (2006). Development and initial validation of a measure of autonomy, competence, and relatedness in exercise: The Basic Psychological Needs in Exercise Scale. Measurement In Physical Education and Exercise Science, 10(3), 179-201.

Worthington, R., \& Whittaker, T. (2006). Scale development research. A content analysis and recommendations for best practices. The Counseling Psychologist, 34(6), 806-838. 


\section{Apêndice}

BPNPES: Basic Psychological Needs in Physical Education Scale

(Questionário de Avaliação das Necessidades Psicológicas Básicas em Educação Física)

Neste questionário, pedimos que nos indiques as tuas experiencias em relação às tuas aulas de Educação Física. Uma vez que não existem respostas certas ou erradas, pedimos que sejas o mais sincero possível. As tuas respostas serão confidenciais e em momento algum serão transmitidas a outras pessoas. Por favor, lê cuidadosamente o questionário e considerando os níveis indicados: 1 "Discordo Totalmente"; 2 "Discordo"; 3 "Não Concordo, Nem Discordo"; 4 "Concordo"; 5 "Concordo Totalmente", responde às seguintes afirmações, colocando um círculo em redor do número que melhor reflecte o teu grau de concordância acerca da mesma.

Na disciplina de Educação Física, geralmente...

1) ...sinto que faço grandes progressos nas minhas aprendizagens

2) ... sinto-me bem com os colegas da minha turma

3) .... forma como faço as actividades está de acordo com as minhas escolhas

4) ....sinto que realizo com sucesso as actividades da aula

5) ...tenho uma relação de amizade com os meus colegas da turma

6) ....sinto que faço as actividades da forma que eu quero

7) ... sinto que faço muito bem as actividades

8) ... sinto que não tenho problemas em relacionar-me com os colegas da minha turma

9) ....as actividades que realizo representam bem aquilo que eu quero fazer

10) ...sou capaz de cumprir com as exigências das actividades da aula

11) ...tenho uma boa relação com os meus colegas da turma

12) ... sinto que tenho oportunidade de escolher a forma como faço as actividades 